\title{
BMJ Open Anonymisation of address coordinates for microlevel analyses of the built environment: a simulation study
}

\author{
Christoph Buck, Steffen Dreger, Iris Pigeot
}

To cite: Buck C, Dreger S, Pigeot I. Anonymisation of address coordinates for microlevel analyses of the built environment: a simulation study. BMJ Open 2015;5:e006481. doi:10.1136/bmjopen-2014006481

- Prepublication history for this paper is available online. To view these files please visit the journal online (http://dx.doi.org/10.1136/ bmjopen-2014-006481).

Received 2 September 2014 Revised 22 January 2015 Accepted 7 February 2015

Leibniz Institute for Prevention Research and Epidemiology-BIPS, Bremen, Germany

Correspondence to Christoph Buck; buck@bips.uni-bremen.de

\section{ABSTRACT}

Background: Data privacy is a major concern in spatial epidemiology because exact residential locations or parts of participants' addresses such as street or zip codes are used to perform geospatial analyses. To overcome this concern, different levels of aggregation such as census districts or zip code areas are mainly used, though any spatial aggregation leads to a loss of spatial variability. For the assessment of urban opportunities for physical activity that was conducted in the IDEFICS (Identification and prevention of dietaryand lifestyle-induced health effects in children and infants) study, macrolevel analyses were performed, but the use of exact residential addresses for microlevel analyses was not permitted by the responsible office for data protection. We therefore implemented a spatial blurring to anonymise address coordinates depending on the underlying population density. Methods: We added a standard Gaussian distributed error to individual address coordinates with the variance $\sigma^{2}$ depending on the population density and on the chosen k-anonymity. 1000 random point locations were generated and repeatedly blurred 100 times to obtain anonymised locations. For each location $1 \mathrm{~km}$ network-dependent neighbourhoods were used to calculate walkability indices. Indices of blurred locations were compared to indices based on their sampling origins to determine the effect of spatial blurring on the assessment of the built environment. Results: Spatial blurring decreased with increasing population density. Similarly, mean differences in walkability indices also decreased with increasing population density. In particular for densely-populated areas with at least 1500 residents per $\mathrm{km}^{2}$, differences between blurred locations and their sampling origins were small and did not affect the assessment of the built environment after spatial blurring.

Conclusions: This approach allowed the investigation of the built environment at a microlevel using individual network-dependent neighbourhoods, while ensuring data protection requirements. Minor influence of spatial blurring on the assessment of walkability was found that slightly affected the assessment of the built environment in sparsely-populated areas.

\section{BACKGROUND}

Data privacy is a major issue in conducting epidemiological studies. In spatial epidemiology,

\section{Strengths and limitations of this study}

Spatial blurring only induces small differences in values of the walkability index between blurred locations and their origins.

- Impact of spatial blurring on the association between walkability and physical activity could not be investigated.

- The use of a large simulated data set enabled us to identify differences in the walkability index and to calculate the k-anonymity of blurred points.

data protection becomes an even more important concern, since the exact residential location or parts of participants' address information such as street names or zip codes are used to perform geospatial analyses. ${ }^{12}$ To overcome this concern different levels of aggregation such as census districts or zip code areas are mainly used for spatial analyses of disease patterns or to assess the built environment in the neighbourhood of participants. ${ }^{3}$ Any administrative division, however, leads to a loss of spatial variability and the use of address proxies based on centroids of aggregated areas induces large positional discrepancies. ${ }^{3-5}$ Considering built environment research, the use of ego-centred neighbourhoods based on the home address of participants is recommended to assess environmental exposure of the built environment on a microlevel and to investigate its influence on physical activity of residents. $^{6-8}$

Complementary to the overall examination programme of the IDEFICS study (Identification and prevention of dietary- induced and lifestyle-induced health effects in children and infants), ${ }^{9}$ we performed environmental analyses in German study regions using geographic information systems (GIS) to investigate the impact of the built environment on the physical activity of children. ${ }^{10}$ The IDEFICS study was conducted from 2006 to 2012 as a longitudinal multicentre study examining 16228 children aged 2-9 years from 
eight European countries at baseline to investigate the aetiology of lifestyle-related and nutrition-related diseases. ${ }^{9}$ In one German study region, pilot analyses of the built environment were conducted on a macrolevel considering school catchment areas as aggregation level to assess the living environment of participating children. ${ }^{10}$

Linking individual and environmental data based on administrative areas has two main disadvantages. First, the use of school catchment areas, which are defined by the municipality, reduces the variability of environmental variables of participants, for example, by calculating average values of these variables in oftentimes large areas. ${ }^{6}$ Second, administrative areas are artificially defined and as such do not necessarily reflect individual characteristics or patterns in physical behaviour. Adjacent school catchment areas, for instance, may capture the living environment of children living near schools, but children living at the border of one area may be influenced by characteristics of an adjacent area, which is referred to as the container effect. ${ }^{4}$ Hence, assessing the environment of participants based on their exact home address provides the best information on the individual living environment. Such an approach may also overcome the modifiable area unit problem which results from artificial areas and varying sizes of aggregation levels. ${ }^{7}$ In the IDEFICS study, however, the use of exact address coordinates of participants was not permitted by the office for data protection of Lower Saxony, which was responsible for the two German study regions.

We therefore considered a geomasking approach to anonymise address data of participants in the German study regions of the IDEFICS study, which provides a simple method to blur participants' address coordinates by adding a standard Gaussian error. ${ }^{2}{ }^{11}$ According to Cassa $e t a l,{ }^{11}$ this approach only slightly affects cluster detection based on spatial scan statistics, but it is not clear how the assessment of built environment measures is affected. ${ }^{6}{ }^{8}$ Thus, we calculated the walkability index according to Freeman et $a l^{12}$ in ego-centred networkdependent neighbourhoods around randomly generated point locations and their repeatedly blurred counterparts. Differences between the value of the index based on sampling origins and based on blurred locations were considered to assess whether this approach has an effect on walkability measures. Hence, the simulation study was conducted to investigate the effect of spatial blurring on walkability measures that are calculated on a microlevel while ensuring data protection requirements.

\section{METHODS}

\section{Spatial blurring}

Implementing a fixed standard Gaussian error in a varying urban environment does not provide a proper disturbance that prevents the reidentification of addresses of participants' home with regard to k-anonymity, where one participant should not be reidentified from at least $\mathrm{k}-1$ individuals. ${ }^{11} 1314$ For example, a fixed disturbance value used for one study region possibly allows to reidentify participants in sparsely-populated subdistricts, because anonymised addresses may be located close to less than $\mathrm{k}$ residents. In densely-populated subdistricts of the study area, such a disturbance value may be too large and could be reduced while still preventing reidentification of participants within $\mathrm{k}$ residents. ${ }^{11}$

To consider the variability of population density within a study region, we implemented a procedure to anonymise given coordinates $a_{i}=\left(b_{i}, c_{i}\right), a_{i} \in R^{2}$, of participants $\mathrm{i}=1, \ldots, \mathrm{n}$ on a surface $\mathrm{W} \subset R^{2}$. In detail, we added a spatial blurring $\mathrm{S}_{\mathrm{i}}=\left(\mathrm{X}_{\mathrm{i}}, \mathrm{Y}_{\mathrm{i}}\right)$ based on independent Gaussian distributed errors $\mathrm{X}_{\mathrm{i}}, \mathrm{Y}_{\mathrm{i}} \sim \mathcal{N}\left(0, \sigma_{\mathrm{i}}^{2}\right)$ for both components to obtain the anonymised coordinates $\mathrm{a}_{\mathrm{i}}+\mathrm{S}_{\mathrm{i}}=\left(\mathrm{b}_{\mathrm{i}}+\mathrm{X}_{\mathrm{i}}, \mathrm{c}_{\mathrm{i}}+\mathrm{Y}_{\mathrm{i}}\right)$, where $\mathrm{S}_{\mathrm{i}} \sim \mathcal{N}\left(0, \sigma_{\mathrm{i}}^{2} \mathrm{I}_{2}\right)$ and $\mathrm{I}_{2}$ is the two-dimensional identity matrix. Anonymised coordinates $\mathrm{a}_{\mathrm{i}}+\mathrm{S}_{\mathrm{i}}$ are located in a $3 \sigma_{\mathrm{i}}$ circle around the original address coordinates with probability of about $99.7 \%$, due to the Gaussian distribution. Hence, we considered the area $|\mathrm{B}|=9 \sigma_{\mathrm{i}}^{2} \pi$ to define the degree of anonymisation using the expected age-specific number $\mathrm{k}_{\sigma}$ of residents that provides k-anonymity given as $\mathrm{k}_{\sigma}=|\mathrm{B}| \mathrm{p}_{\mathrm{A}} \mathrm{RD}_{\mathrm{A}}$, where $\mathrm{RD}_{\mathrm{A}}$ is the number of residents per $\mathrm{km}^{2}$ and $\mathrm{p}_{\mathrm{A}} \in[0,1]$ is the age-specific percentage of residents in a given subdistrict $\mathrm{A} \subset \mathrm{W}$. Based on the parameter of k-anonymity $\mathrm{k}_{\sigma}$, we derived the variance $\sigma_{\mathrm{i}}^{2}$ of the spatial blurring as being inversely proportional to the underlying population density $\mathrm{p}_{\mathrm{A}} \cdot \mathrm{RD}_{\mathrm{A}}$ of the specific age group as

$$
\sigma_{\mathrm{i}}^{2}(\mathrm{~A})=\frac{\mathrm{k}_{\sigma}}{9 \pi \cdot \mathrm{p}_{\mathrm{A}} \cdot \mathrm{RD}_{\mathrm{A}}} .
$$

In summary, the variance of the Gaussian error will be calculated for any coordinate based on the underlying population density of the considered age group and the chosen k-anonymity. The blurring is then induced by the inverse Gaussian distribution with mean value of zero and the given variance and is added to the original coordinates. Coordinates in densely-populated areas will be blurred less, and vice versa.

\section{Built environment measures}

Spatial data on land use and population density were provided by the land registry office of Lower Saxony. Data on footpath network were obtained from the OpenStreetMap project (http://www.openstreetmap. org) and validated using official data. Additionally, bus stops were digitalised based on available maps and lists provided by the public transit company. ${ }^{10}$ ArcGIS 10.0 (ESRI 2011. ArcGIS Desktop: Release 10. Redlands) was used to process and digitalise spatial data as well as to generate random locations within the study region.

The walkability index was calculated based on egocentred network-dependent neighbourhoods capturing an area that can be reached within $1 \mathrm{~km}$ based on the street network. ${ }^{6}{ }^{8}$ For both, circular and networkdependent neighbourhoods, the appropriate size of 
buffers that should be used to assess built environment measures is still discussed in the literature. Commonly used buffer distances range from 400 to $3200 \mathrm{~m}$ and are mainly defined according to walking distances. ${ }^{7}$ Thus, we determined the distance of $1 \mathrm{~km}$ based on a 10 to 15 min walking distance. Network-dependent neighbourhoods around blurred points and their origins were calculated using the network analyst-tool in ArcGIS 10.0.

Walkability measures were calculated within these egocentred neighbourhoods using the spatstat-package $(1.33 .0)^{15}$ in $R(3.0 .1):^{16}$

- Number of residents per $\mathrm{km}^{2}$,

- Land use mix based on the entropy of land use types,

- Number of intersections per area to assess the street connectivity,

- And number of bus stops per area to assess public transit density.

To standardise all four measures, z-scores of each variable were calculated based on mean and SD of walkability measures determined separately for sampling origins and for each blurring step. Z-scores of all four measures were then summed up to the walkability index according to Freeman et al. ${ }^{12}$ Thus, the walkability score is a standardised non-dimensional score with mean value 0 and a SD of 4 based on the four standardised components.

\section{Study data}

We focussed our analyses of the built environment on one German survey region of the IDEFICS study to validate the approach of spatial blurring. ${ }^{10}$ For this purpose, we thus considered built environment measures that were assessed in the city of Delmenhorst, Lower Saxony, which covers an area of about $62 \mathrm{~km}^{2}$ with about 77300 residents. The baseline survey of the IDEFICS study was conducted in 2007/2008 and geographical data were assessed for the same year. ${ }^{9}$

We generated 1000 random locations $i=1, \ldots, 1000$, based on the underlying population density of the age-group that was eligible for the IDEFICS study within subdistricts in the study area of Delmenhorst which are presented in figure 1 . We performed the spatial blurring based on the presented approach using data on residential density and the age-specific percentage of children below the age of 12 per subdistricts. These data were provided by the municipality of Delmenhorst. Randomly generated sampling origins $i=1, \ldots, 1000$ were repeatedly blurred 100 times, $j=1, \ldots, 100$, based on the underlying age-specific population density $\mathrm{p}_{\mathrm{A}} \cdot \mathrm{RD}_{\mathrm{A}}$ and a given k-anonymity $\mathrm{k}_{\sigma}$ using the spatstat-package (1.33.0) and the $s p$-package (1.0-13) in $\mathrm{R}$ (3.0.1).

With regard to statistics of aggregated areas, thresholds for k-anonymity of 5 or 10 are commonly used to prevent the reidentification of participants. ${ }^{13}{ }^{14}$ However, determining k-anonymity for spatial analyses leads to a trade-off between geomasking of addresses and resulting k-anonymity, which has significant computational challenges. ${ }^{14}{ }^{17}$ To identify the best approach that provides a compromise between geomasking and k-anonymity, we blurred sampling origins considering values of $\mathrm{k}_{\sigma}=10,15$ as a parameter for the blurring variance $\sigma_{\mathrm{i}}^{2}$. By this, we assumed that our virtual participants should not be reidentified among 10 and 15 residents of the same age group, respectively. We chose values greater than $\mathrm{k}=5$ as parameter for k-anonymity, since shifting original addresses of participants through spatial blurring can result in anonymised locations that fall into sparsely-populated areas where common thresholds of k-anonymity may not be fulfilled anymore. We therefore calculated the observed k-anonymity $\hat{k}_{i, j}$ of blurred locations $\mathrm{j}=1, \ldots, 100$ and origins $\mathrm{i}=1, \ldots, 1000$ for each parameter $\mathrm{k}_{\sigma}$ based on the underlying age-specific population density within $3 \sigma_{\mathrm{i}}$ circles and evaluated if the observed k-anonymity $\hat{k}_{i, j}$ did not fall under a threshold of $\mathrm{k}=5$.

\section{Statistical analyses}

To investigate the variation of the simulated blurring, mean, SD, and range of the simulated blurring $\left|\mathrm{s}_{\mathrm{i}}\right|=\sqrt{\mathrm{x}_{\mathrm{i}}^{2}+\mathrm{y}_{\mathrm{i}}^{2}}$ were calculated for each sampling origin. Additionally, the mean, SD, and range of $\hat{k}_{i, j}$ of blurred locations were also calculated for each sampling origin. Spatial blurring and $\hat{k}_{i, j}$ were depicted depending on the underlying population density. The walkability index was calculated based on ego-centred neighbourhoods of 1000 sampling origins $\mathrm{i}$ and of 100000 blurred locations $\mathrm{j}$. Owing to the spatial blurring, locations fell outside the study area and neighbourhoods could not be calculated for $480\left(\mathrm{k}_{\sigma}=10\right)$ and $476\left(\mathrm{k}_{\sigma}=15\right)$ blurred locations. Differences in values of the walkability index between blurred locations and their related origins were calculated to assess changes in the index that were induced by spatial blurring. For each sampling origin i mean differences of walkability indices of blurred locations $\mathrm{j}$ to the related origin were calculated and displayed depending on the underlying population density. All statistics were calculated in SAS 9.3 (SAS Institute Inc., Cary, North Carolina, USA).

\section{RESULTS}

Figure 2 presents statistics of the simulated blurring $\left|\mathrm{s}_{\mathrm{i}}\right|$ and the resulting $\hat{k}_{i, j}$ compared to the initial $\sigma_{\mathrm{i}}$ and the threshold of $\mathrm{k}=5$, respectively. Mean shift of $\left|\mathrm{s}_{\mathrm{i}}\right|$ is close to the initial variance $\sigma_{\mathrm{i}}$ for both values of $\mathrm{k}_{\sigma}$. Spatial blurring induced higher values of about 200-500 $\mathrm{m}$ for areas with less than 1000 residents per $\mathrm{km}^{2}\left(\mathrm{k}_{\sigma}=10\right)$ and for areas with less than 1500 residents per $\mathrm{km}^{2}\left(\mathrm{k}_{\sigma}=15\right)$, respectively. Accordingly, mean and maximum shift $\left|\mathrm{s}_{\mathrm{i}}\right|$ decreased for densely-populated areas with more than 1500 residents per $\mathrm{km}^{2}$. The maximum shift $\left|\mathrm{s}_{\mathrm{i}}\right|$ showed values of $150 \mathrm{~m}\left(\mathrm{k}_{\sigma}=10\right)$ or $200 \mathrm{~m}\left(\mathrm{k}_{\sigma}=15\right)$ at most, while mean $\left|\mathrm{s}_{\mathrm{i}}\right|$ decreased from 48 to $32 \mathrm{~m}\left(\mathrm{k}_{\sigma}=10\right)$ and 58 to $39 \mathrm{~m} \quad\left(\mathrm{k}_{\sigma}=15\right)$ (figure 2, top). Summing up, for both values of k-anonymity the mean and maximum shift decreased with increasing residential density. Assuming a higher value of $\mathrm{k}$-anonymity $\left(\mathrm{k}_{\sigma}=15\right)$, that is a participant 
Figure 1 Distribution of random points and age-specific population density of subdistricts in the study region Delmenhorst, Germany.

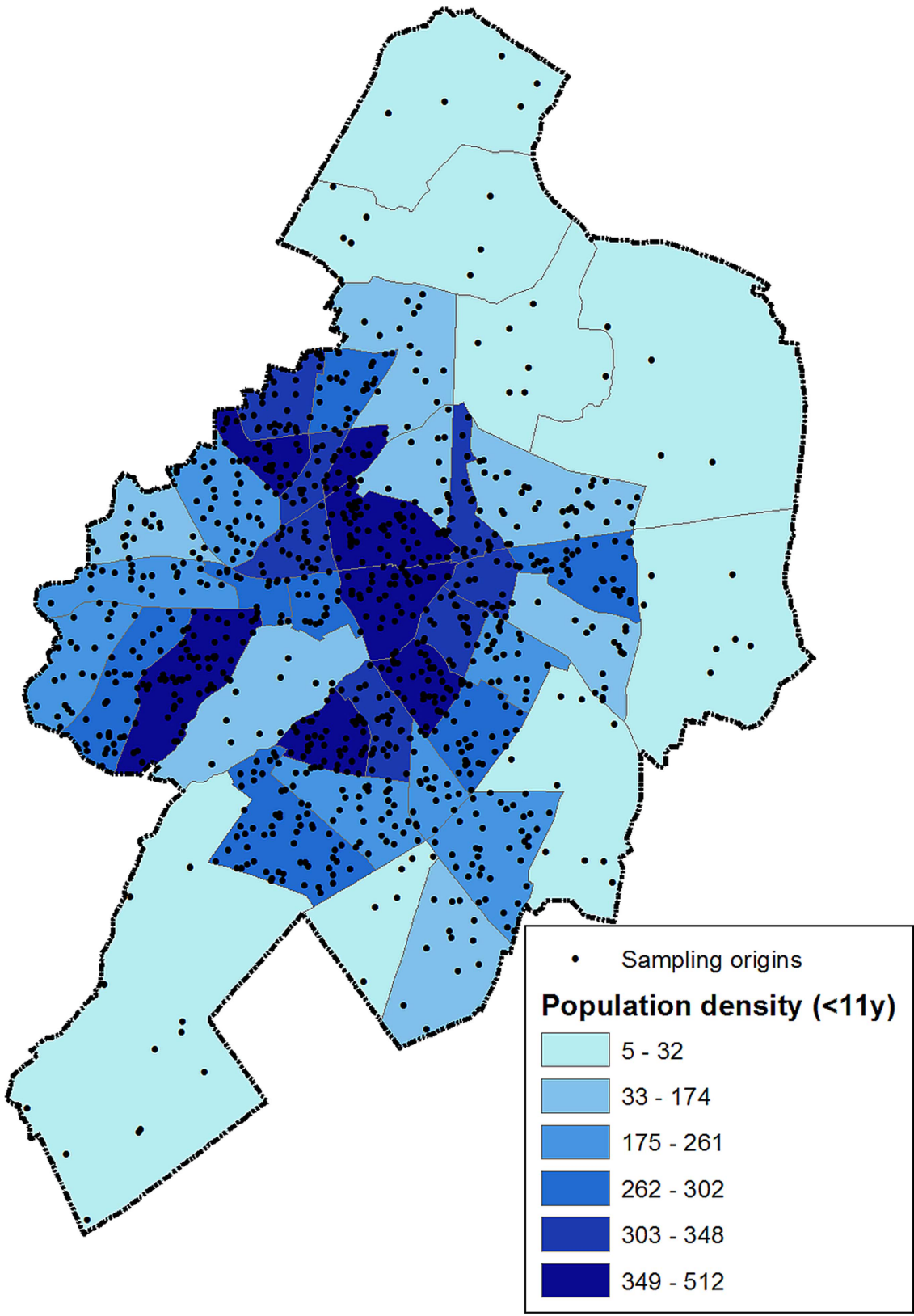

cannot be reidentified from at least 15 residents, the shift of the coordinates was slightly higher compared to the lower value of k-anonymity.

Mean observed k-anonymity $\hat{k}_{i, j}$ leveled off at the chosen parameter $\mathrm{k}_{\sigma}$. On the basis of higher value of k-anonymity $\mathrm{k}_{\sigma}=15$, the observed $\hat{k}_{i, j}$ was higher than based on the lower value $\mathrm{k}_{\sigma}=10$ (figure 2, bottom). For both parameters, the observed k-anonymity $\hat{k}_{i, j}$ showed values below the threshold of $\mathrm{k}=5$ and even reached zero. Overall, the observed k-anonymity $\hat{k}_{i, j}$ fell below $\mathrm{k}=5$ for $4.3 \% \quad\left(\mathrm{k}_{\sigma}=10\right)$ and $1.2 \% \quad\left(\mathrm{k}_{\sigma}=15\right)$ of blurred address locations, respectively. In other words, on average the assumed parameter of k-anonymity was met. However, assuming the less restrictive k-anonymity $\left(\mathrm{k}_{\sigma}=10\right.$ compared to $\left.\mathrm{k}_{\sigma}=15\right)$, the blurred coordinates were more likely to be reidentified from less than five residents after spatial blurring.
Values of walkability indices of 1000 original locations $\mathrm{i}$ showed mean and SD of $0.92 \cdot 10^{-15}$ and 2.9 and ranged from -10.5 to 6.6. Figure 3 shows mean differences in the walkability indices between blurred locations $j$ and their related sampling origins $i$ depending on the underlying population density $\mathrm{RD}_{\mathrm{A}}$. Each dot shows the mean difference between the walkability index based on the neighbourhood of the sampling origin and walkability indices based on the neighbourhoods of 100 blurred locations of this origin. For both values of $\mathrm{k}$-anonymity $\mathrm{k}_{\sigma}$, the mean difference strongly decreased for a higher population density and did not exceed a value of 1 for at least 1000 residents per $\mathrm{km}^{2}$ with only some exceptions. Summarising, it became obvious that the walkability index remained rather stable when shifting the address coordinates in densely-populated areas 

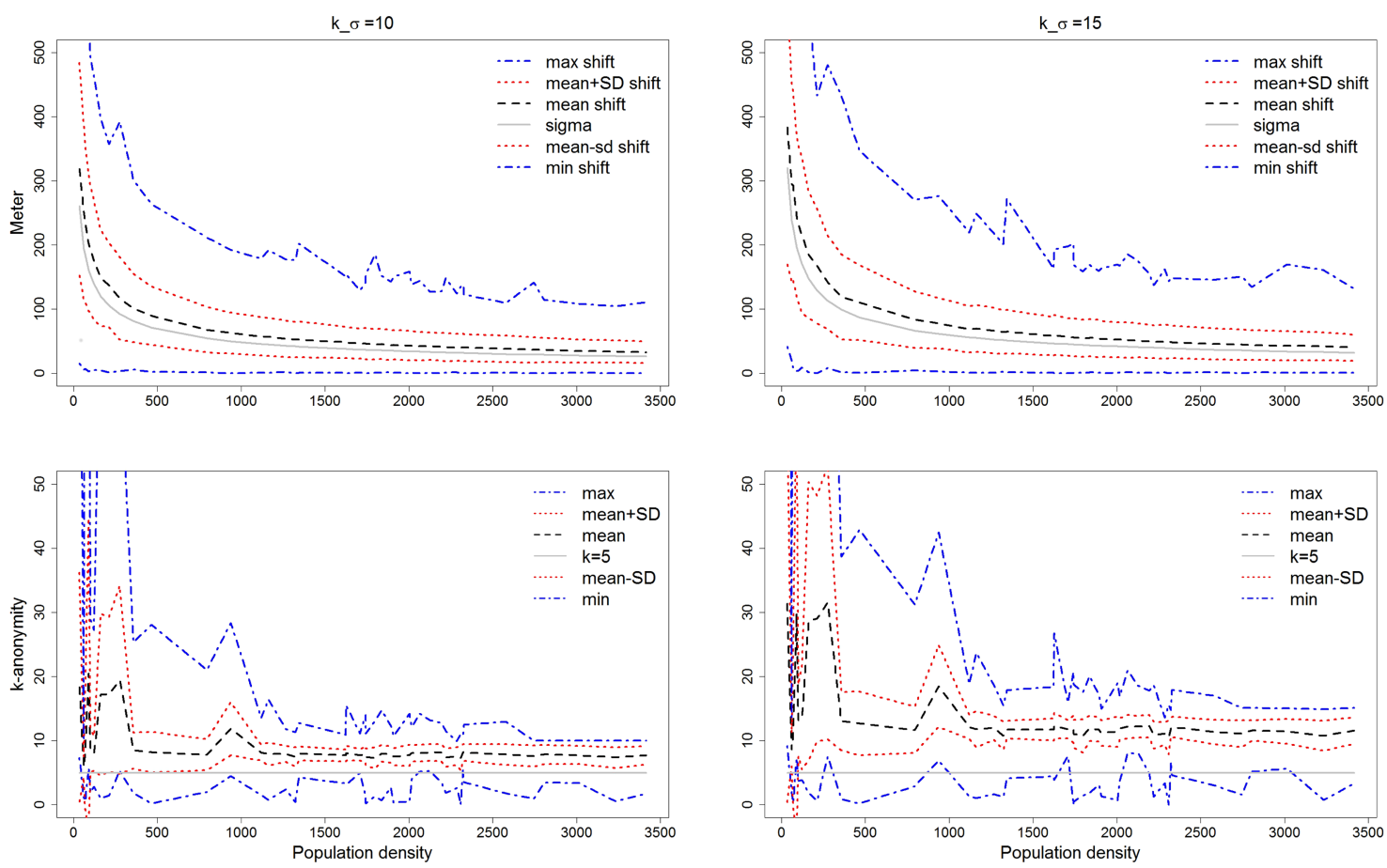

Figure $2 \mathrm{SD} \sigma_{\mathrm{i}}$ of the spatial blurring $\left|\mathrm{S}_{\mathrm{i}}\right|$ and summary statistics of the simulated spatial blurring $\left|\mathrm{s}_{\mathrm{i}}\right|$ (top) and of resulting $\hat{k}_{i, j}$ (bottom) depending on the underlying population density $\mathrm{RD}_{\mathrm{A}}$ and two parameters $\mathrm{k}_{\sigma}=10,15$.

whereas it showed some more variability in sparselypopulated areas. For the more restrictive k-anonymity, a slightly higher difference between the walkability indices based on the blurred locations and the original location was observed.

\section{DISCUSSION}

The simulated spatial blurring that we conducted was based on randomly generated locations. In denselypopulated areas, it resulted in only small shifts for most original locations. Owing to the population-dependent $\sigma_{\mathrm{i}}$, the blurred points were rather strongly shifted in sparsely-populated areas, but the shift was less than $50 \mathrm{~m}$ on average for densely-populated areas with at least 1500 residents per $\mathrm{km}^{2}$. Apparently, a stronger shift was observed for $\mathrm{k}_{\sigma}=15$ than for $\mathrm{k}_{\sigma}=10$, whereas for $\mathrm{k}_{\sigma}=15$ the resulting k-anonymity $\hat{k}_{i, j}$ fell under the threshold of $\mathrm{k}=5$ only in $1.2 \%$ of blurred locations.

Mean differences between walkability indices based on blurred points and on their related sampling origins showed similar patterns. For sparsely-populated areas, spatial blurring in most cases had a stronger impact on the walkability index. However, for blurred locations in densely-populated areas with at least 1000 residents per $\mathrm{km}^{2}$, spatial blurring only led to differences in the walkability index of about $0.5-1$ at most for both values of $\mathrm{k}_{\sigma}=10,15$. Compared to the range of the walkability index of original locations, these differences were acceptable and only slightly affected the assessment of built environment measures. On the basis of this approach, the use of anonymised individual coordinates of the home address was allowed by the office for data protection of Lower Saxony. In summary, we can
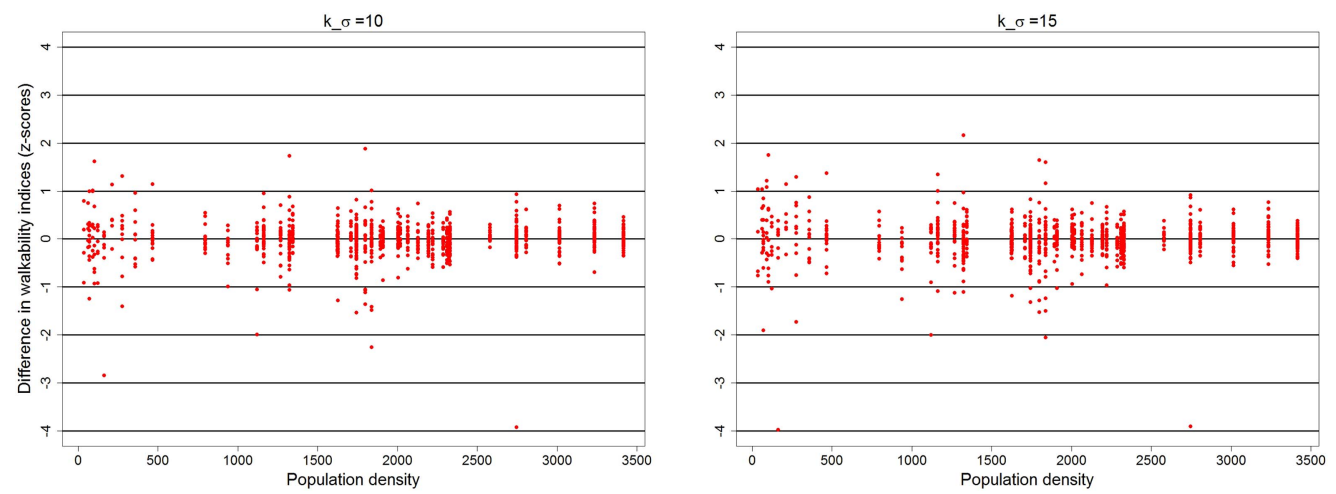

Figure 3 Mean difference in walkability indices between original coordinates and blurred coordinates (dots) depending on the underlying population density $\mathrm{RD}_{\mathrm{A}}$ for parameters $\mathrm{k}_{\sigma}=10,15$. 
therefore recommend $\mathrm{k}_{\sigma}=15$ as a reasonable compromise between an acceptable shift and k-anonymity. Based on the SD of walkability measures in our study, a difference of 0.5 is equivalent to $17 \%$ of the SD. In larger study regions with higher variation in built environment characteristics, the walkability index can show a higher range, as for example in Freeman et al, ${ }^{12}$ where the index showed values from -7.9 to 11.7. Thus, the effect of spatial blurring might be even lower depending on the size and environmental variability of the study area.

Our findings concerning the assessment of built environment measures are similar to the results of Cassa et $a l^{11}$ who showed that the spatial blurring only slightly affected cluster detection analyses. However, some limitations have to be discussed. Cassa $e t a l^{18}$ deduced a vulnerability of spatial blurring which may enable adversaries to reidentify individuals based on the mean coordinates of multiple anonymised versions of the original data. Deriving ego-centred neighbourhoods based on address coordinates, that were only blurred once, should reduce the risk of reidentification of study participants.

Sparsely-populated areas, such as parts in the centre of the study area, induced larger changes in the walkability index if these areas were adjacent to denselypopulated areas. Different approaches, such as the donut method, ${ }^{19}$ may reduce the geomasking error and should restrict effects of sparsely-populated areas within or at the edge of the study area as long as thresholds for k-anonymity are maintained.

Using a large simulated data set is the major strength of our study, since the knowledge about the origins and the outcome of our approach enabled us to identify the difference in the walkability index and to calculate the k-anonymity of blurred points. Repeating the spatial blurring multiple times for one origin also controlled for the variation in our results.

However, consequences for real study samples have to be further investigated. In the IDEFICS study, the majority of the participating children lived in urban and densely-populated areas of the study region. ${ }^{10}$ Thus, the spatial blurring should not affect the investigation of the built environment in the majority of the study sample. Rural and sparsely-populated areas may have an effect on the assessment of the built environment, due to the changes in walkability indices of blurred locations that were found in our analyses. Excluding participants that live in areas with less than 1000 residents per $\mathrm{km}^{2}$ should ensure that spatial blurring does not lead to an undesired bias in the walkability index. However, the association of physical activity and built environment might then again be affected due to differences in the physical behaviour between residents of urban and rural neighbourhoods. Thus, the exclusion of participants in sparsely-populated areas still needs to be further investigated.

Eventually, we only inferred the effect of spatial blurring on the assessment of built environment measures, but not on the association between physical activity levels of residents and walkability in their neighbourhood. Thus, further analyses based on data of participants whose addresses are allowed to be used in spatial analyses are necessary to determine the effect of spatial blurring on the association between built environment and physical activity levels.

Taking the strengths and limitations into account, the effect of spatial blurring on built environment measures and on the walkability index was found to be relatively small. This supports the feasibility of the presented geomasking approach for densely-populated areas with at least 1000 residents per $\mathrm{km}^{2}$. The use of point locations to assess the built environment on a micro-level is a strong advantage compared to the use of administrative areas and may compensate both, the disadvantages of spatial blurring and of a potential exclusion bias of participants that live in sparsely-populated areas. ${ }^{7} 8$ Investigating the association between physical activity levels of residents and the walkability of their neighbourhood, we may therefore conclude that a small change in the walkability index may only slightly influence the assessment of built environment measures.

\section{CONCLUSION}

The presented approach allows to assess the built environment on a microlevel while ensuring data protection requirements. It facilitates to determine the urban neighbourhood of each participant using ego-centred network-dependent neighbourhoods based on anonymised address coordinates. Small differences were found in the walkability index which should only slightly affect further assessment of the built environment. As a result of the spatial blurring, available individual contact details, such as address information, can be used to assess environmental exposure combining individual and environmental data on a microlevel while ensuring data protection requirements.

Acknowledgements The authors acknowledge the cooperation with the office for data protection of Lower Saxony, Germany.

Contributors IP, SD, and CB conceptualised the research question and the geospatial analyses and interpreted the results. IP and CB discussed the results with the responsible office for data protection. $\mathrm{CB}$ conducted the analyses and wrote the manuscript and SD and IP reviewed the manuscript. All authors approved the final manuscript for publication.

Funding This work was funded by the German Research Foundation (DFG) under grant PI 345/7-1.

\section{Competing interests None.}

Provenance and peer review Not commissioned; externally peer reviewed.

Data sharing statement The $\mathrm{R}$ code and the simulated data is available from the authors on request

Open Access This is an Open Access article distributed in accordance with the Creative Commons Attribution Non Commercial (CC BY-NC 4.0) license, which permits others to distribute, remix, adapt, build upon this work noncommercially, and license their derivative works on different terms, provided the original work is properly cited and the use is non-commercial. See: http:// creativecommons.org/licenses/by-nc/4.0/ 


\section{REFERENCES}

1. Olson KL, Grannis SJ, Mandl KD. Privacy protection versus cluster detection in spatial epidemiology. Am J Public Health 2006;96:2002-8.

2. Boulos MN, Curtis AJ, Abdelmalik P. Musings on privacy issues in health research involving disaggregate geographic data about individuals. Int J Health Geogr 2009;8:46-53.

3. Matisziw TC, Grubesic TH, Wie H. Downscaling spatial structure for the analysis of epidemiological data. Comput Environ Urban Syst 2008;32:81-93.

4. Maroko AR, Maantay JA, Sohler NL, et al. The complexities of measuring access to parks and physical activity sites in New York City: a quantitative and qualitative approach. Int $J$ Health Geogr 2009;8:34-56.

5. Healy MA, Gilliland JA. Quantifying the magnitude of environmental exposure misclassification when using imprecise address proxies in public health research. Spat Spatiotemporal Epidemiol 2012;3:55-67.

6. Frank LD, Schmid TL, Sallis JF, et al. Linking objectively measured physical activity with objectively measured urban form: findings from SMARTRAQ. Am J Prev Med 2005;28(2 Suppl 2):117-25.

7. Brownson RC, Hoehner CM, Day K, et al. Measuring the built environment for physical activity: state of the science. Am J Prev Med 2009;36(4 Suppl):S99-123.

8. Perchoux $\mathrm{C}$, Chaix B, Cummins $\mathrm{S}$, et al. Conceptualization and measurement of environmental exposure in epidemiology: accounting for activity space related to daily mobility. Health Place 2013;21:86-93.

9. Ahrens W, Bammann K, Siani A, et al. The IDEFICS cohort: design, characteristics and participation in the baseline survey. Int $J$ Obes 2011;35(Suppl 1):S3-15.
10. Buck C, Pohlabeln H, Huybrechts I, et al. Development and application of a moveability index to quantify possibilities for physical activity in the built environment of children. Health Place 2011;17:1191-201.

11. Cassa CA, Grannis SJ, Overhage JM, et al. A contextsensitive approach to anonymizing spatial surveillance data: impact on outbreak detection. J Am Med Informatics Assoc 2006;13:160-5.

12. Freeman L, Neckerman K, Schwartz-Soicher O, et al. Neighborhood walkability and active travel (walking and cycling) in New York City. $J$ Urban Health 2013;90:575-85.

13. Sweeney L, K-anonimity: a model for protecting privacy. Int $J$ Uncertain Fuzziness Based Syst 2002;10:557-70.

14. Bayardo RJ, Agrawal R. Data privacy through optimal k-anonymization. Proceedings-International Conference on Data Engineering; 2005:217-28.

15. Baddeley A, Turner R. Modelling spatial point patterns in R. Case Stud Spat point Process Model 2006:1-51.

16. R Core Team. R: A language and environment for statistical computing. R Foundation for Statistical Computing, Vienna, Austria, 2014. http://www.R-project.org/

17. Meyerson A, Williams R. On the complexity of optimal K-anonymity. Proceedings of the twentythird ACM SIGMOD-SIGACT-SIGART Symposium on Principles of Database systems PODS 04; 2004:223-8.

18. Cassa CA, Wieland SC, MandI KD. Re-identification of home addresses from spatial locations anonymised by Gaussian skew. Int J Health Geogr 2008;7:45-53.

19. Hampton KH, Fitch MK, Allshouse WB, et al. Mapping health data: improved privacy protection with donut method geomasking. $A m \mathrm{~J}$ Epidemiol 2010;172:1062-9. 